\title{
Investigation the effect of oral Aloe Vera gel pills supplementation on the intensity of
} primary menstrual pain (Dysmenorrhea)

\author{
Samaneh Sardashti ${ }^{1,2}$, Tahere Sarboozi Hosein Abadi ${ }^{1,2}$, Shoaib Sarboozi Hosein Abadi ${ }^{3}$, \\ Rasool Raznahan ${ }^{1,2^{*}}$
}

Corresponding author: Rasool Raznahan, E-mail: rr4239@gmail.com

1- Department of Nursing, School of Nursing and Midwifery, Torbat Heydariyeh University of Medical Sciences,

Torbat Heydariyeh, Iran

2 - Health Sciences Research Center, Torbat Heydariyeh University of Medical Sciences, Torbat Heydariyeh, Iran.

3 - Department of Emergency Nursing, School of Nursing and Midwifery, Birjand University of Medical Sciences,

\section{Abstract}

Birjand, Iran

Introduction. This study was conducted to evaluate the effects of oral aloe vera gel pills supplementation on the intensity of menstrual pain. A randomized, single-blind clinical experimental plan was conducted amongst 150 single students aged 20 to 26 years who suffered from menstrual pain, at the Islamic Azad University of Medical Sciences from December 24, 2015 to October 22, 2016. The extent of pain was evaluated using the Cox Menstrual Symptom Scale (CMSC).

Material and method. Each participant was randomly assigned to aloe vera gel pill or the anti-PG drug Ibuprofen, ending with 60 participants in each group equally. The first trial group received $10 \mathrm{mg}$ of aloe vera gel orally four times daily until the pain grade reached one or less. The control group received Ibuprofen and three tablets orally three times a day. The participants were permitted to take another drug that they usually took for their pain relief, in addition to the allocated treatment in case of continued pain. At the end of the trial, these participants were excluded in data analysis.

Results and discussions. Changes in the grade and the duration of the pain of participants were compared at the first and second months in both groups. Sample size was computed using $\alpha=5 \%$ and absolute error equal to 0.24 for correlation between medication and pain with acceptable absolute precision formula (AAPF). Result of this study showed that the mean age was $22.1 \pm 1.4$ and $20.4 \pm 1.2$ years in aloe vera gel pills and Ibuprofen groups, respectively. No significant difference was observed for the matched characteristics studied between the treated groups. the mean pain grade amongst aloe vera group was decreased from $2.81 \pm 0.65$ to $2.02 \pm 0.34$, while in Ibuprofen group from $2.48 \pm 0.4$ to $1.32 \pm 0.15$, respectively, using the described given dose. As result obtained that there was no statistically significant decrease in pain duration for the women who received Ibuprofen compared to those who used aloe vera. Also there was no significant difference in pain grade between the groups at the second month of intervention $(\mathrm{p}=0.61)$. Pain duration at the second month of trial was similar between the both groups $(\mathrm{p}=0.76)$.

Conclusions. In conclusion we could suggest that aloe vera gel pill as a novel herbal pain killer represents an effective treatment for the menstrual pain with no important side effects, although further clinical trials are recommended to look at the possible side effects in an extended spectrum of subjects.

Key words: Aloe vera gel, Dysmenorrhea, Menstrual pain, Medicine, Symptoms, Women,

\section{Introduction}

Primary dysmenorrhea "Painful Periods" is a very common problem in young women. It is usually defined as cramping pain in the lower abdomen occurring at the onset of menstruation in the absence of any identifiable pelvic disease. A pain which is of uterine origin and directly linked to menstruation but with no visible pelvic pathology is called primary dysmenorrhea. and is distinguished from secondary dysmenorrhea, which refers to painful menses resulting from pelvic pathology such as endometriosis. It usually presents during adolescence, within three years of menarche. It is unusual for symptoms to start within the first six months after menarche. Affected women and young girls experience sharp, intermittent spasms of pain, usually centered in the suprapubic area. Pain may radiate to the back of the legs or the lower back. Additionally, systemic symptoms of nausea, vomiting, diarrhea, fatigue, fever, headache or lightheadedness are fairly common. 
Pain usually develops within hours of the start of menstruation and peaks as the flow becomes heaviest during the first day or two of the cycle. Population surveys suggest a wide variation in prevalence rate of dysmenorrhea from studies around the world including Iran reporting a range between $35 \%$ and $75 \%$. To regulate uterine contractions and uterine tone, many effective drugs and regimens are described before and various studies have also been conducted, but a proper statistical analysis and interpretation are not available. Use of natural substances with therapeutic properties is not new but it has been used since ancient times. Nowadays, a number of drugs prescribed originate from plants and some natural precursors (19). Herbal and dietary therapies are especially suitable for treatment of disorders such as dysmenorrhoea, as they can be self-administered and are often easily available from pharmacies. A recent review of some trials evaluating the efficacy of herbal and dietary therapies in primary and secondary dysmenorrhoea showed that vitamin B1 taken daily may be an effective treatment for dysmenorrhoea. Sun et al., (2009) showed that the obtained pain scores after the treatment and few months of post-treatment in herbal group were significantly lower than those in the control group in women exposed to the dysmenorrhoe . Aloe Vera is a plant that belongs to Liliaceae family that grows easily in hot and arid regions (21). Te existing mucilage tissue at the center of leaves in this plant that is also so-called aloe gel is used for various cosmetics and medical applications. The peripheral leaf cells in this plant produce bitter and yellow color latex that is called aloes. It is composed of anthracene hydroxyl derivatives including aloins $\mathrm{A}$ and B2 and derivatives such as A, B2, and C aloe resins. The other compounds are sugars such as glucose, mannose, and cellulose (18). It has various vitamins consisting of $\mathrm{B} 1, \mathrm{~B} 2, \mathrm{~B} 6, \mathrm{C}, \mathrm{E}$, and folic acid, and minerals such as calcium, sodium, magnesium, zinc, copper, and chrome. Aloe vera is an herbal plant used in a variety of medical conditions such as wounds healing and decrease tissue damages and the ancient Egyptians used Aloe vera for treatment of wounds, burnings, and infections for the first time. Additionally, the other nationalities had used this herbal by various techniques for several purposes. Some researchers demonstrated that aloe vera is one of the effective cures for dysmenorrhea as it treats the menstrual cramps substantially. Some researchers showed that the Indian aloe is a stimulant of the uterus. Hence it is given in cases of painful menstruation. It possesses compound known as aloes which effectively promotes the sterility and treats disturbed menstrual activity and pain in women. Due to the possible side effects of synthetic drugs, there are many attempts for alternatives traditional or herbal treatments. Many evidences have reported that nutrition and metabolism may play an important role in the cause and treatment of menstrual disorders. Keeping this in view, this study was carried out to investigate the effect of different level of oral Aloe Vera gel supplementation on the intensity of primary dysmenorrhea in young women and teenage girls who studied in the Islamic Azad University of Medical Sciences, Iran.

\section{Materials and Methods Study Procedures}

A randomized, single-blind clinical experimental plan was conducted amongst 150 single students aged 20 to 26 years who suffered from menstrual pain, at the Islamic Azad University of Medical Sciences from December 24, 2015 to October 22, 2016. Participants who were single, suffered from menstrual pain, accommodated at the campus of University of Medical Sciences and had no pathological disorders were included in this study. The eligible participants fulfilled the self-completed questionnaire and the scale form and were visited physically by a licensed gynecologist before randomization.

\section{Experimental design}

The extent of pain was evaluated using the Cox Menstrual Symptom Scale1. Each participant was randomly assigned to aloe vera gel pill or the antiPG drug Ibuprofen, ending with 60 participants in each group equally. The first trial group received 10 mg of aloe vera gel orally four times daily until the pain grade reached one or less. The control group received Ibuprofen and three tablets orally three times a day. The participants were permitted to take another drug that they usually took for their pain relief, in addition to the allocated treatment in case of continued pain. However, at the end of the trial,

\footnotetext{
${ }^{1}$-(having no pain $\leq 0$, for $\geq 0.5 \mathrm{~h} \leq 1$, for $0.5-1 \mathrm{~h} \leq 2$, for several hours $\leq 3$ and several days $\leq 4$ ).
} 
these participants were excluded in data analysis. Changes in the grade and the duration of the pain of participants were compared at the first and second months in both groups. A two sectioned questionnaire was used to collect the data. The first section included the demographic data, menstrual history, smoking, diet, exercise and past medical and reproductive history that was completed before the intervention of the trial. The second section was designed to cover the grade and duration of pain and the accompanying symptoms was completed during the two months follow up of the study. The primary outcome was the intensity of menstrual pain, which was determined using the verbal multidimensional scoring System described by (1) and it has 4 grade including grade 0 : menstruation is not painful and daily activity is unaffected, grade 1: Menstruation is painful but seldom inhibits normal activity, analgesics are seldom required; mild pain, grade 2 : daily activity is affected, analgesics required and give sufficient relief so that absence from school is unusual; moderate pain and grade 3: activity clearly inhibited, poor effect of analgesics, vegetative symptoms such as headache, fatigue, vomiting, and diarrhea.

\section{Statistical analysis}

Sample size was computed using $\alpha=5 \%$ and absolute error equal to 0.24 for correlation between medication and pain with acceptable absolute precision formula. A p-value of 0.05 was considered statistically significant. Randomization was determined on a 1:1 basis using random number tables. Statistical comparisons were determined using the Mann-Whitney U test, unpaired t-test, and within-group comparisons were analyzed by paired $t$ test or Wilcox on.

\section{Ethical evaluation}

Participation in the study was voluntary and the participants were free to withdraw from the study whenever they wished. An informed consent was obtained from all participants before enrolment into the study.

\section{Results and discussion}

As result showed that on table 1, the mean age was $22.1 \pm 1.4$ and $20.4 \pm 1.2$ years in aloe vera gel pills and Ibuprofen groups, respectively. No significant difference was observed for the matched characteristics studied between the treated groups.
In term of educational field of study, the higher frequency was seen for the individuals with bachelor science degree. Level in paramedical science (42 out of 100 individuals or $42 \%$ ) compared to the other groups and in term of educational degree totally. Moreover, 71 out of 100 had bachelor science degree in all medical sciences $(71.00 \%)$, including medical students who only were 20 out of 100 $(20 \%)$. Chemical medication was the most common method used by the participants in both groups as the pain relief procedure before interventions applied by the current clinical trial. The subjects were followed up at least for two sequential periodic cycles. The pain grades were similar in both groups before intervention. About $3 \mathrm{~h}$ after the intervention at the first day of menstruation the mean pain grade amongst aloe vera group was decreased from $2.81 \pm 0.65$ to $2.02 \pm 0.34$, while in Ibuprofen group from $2.48 \pm 0.4$ to $1.32 \pm 0.15$, respectively, using the described given dose.

The pain grade was 1 and higher before intervention in both groups, while it reached zero in about $20 \%$ of the individuals of aloe vera group and $17 \%$ of those in Ibuprofen group $6 \mathrm{~h}$ after the intervention so that they did not ask any more interventions (Table 2).

The comparison of the pain duration between the two groups at the first month of the intervention is shown in Table 3. There was no statistically significant decrease in pain duration for the women who received Ibuprofen compared to those who used aloe vera. Also there was no significant difference in pain grade between the groups at the second month of intervention $(p=0.61)$. Pain duration at the second month of trial was similar between the both

groups $(p=0.76)$. Furthermore, the duration of menstrual flow was similar between the two groups before intervention, while Ibuprofen reduced the duration of menstruation compared to the aloe vera at the first and the second month of the intervention. The present results suggested that the both aloe vera and Ibuprofen group had equivalently reduced the grade and the duration of menstrual pain. The effects of aloe vera as an herbal pain killer can be attributed to the reduction of PG synthesis by its action as an antispasmodic and anti-PG. Studies show that $\beta$ adrenoreceptors activation in uterus causes relaxation (8) and a stimulatory effect of $Z$. multiflora Boiss extract has been shown on $\beta 2$ adrenoceptors, which is perhaps due to its 
constituent, carvacrol (Boskabady et al., 2006). Ozgoli et al., (2009) (13) mentioned that there were not significant differences between groups in baseline characteristics, $p>0.05$. At the end of treatment, severity of dysmenorrhea decreased in all groups and no differences were found between the groups in severity of dysmenorrhea, pain relief, or satisfaction with the treatment, $p>0.05$. No severe side effects occurred. Khazaiyan and Navidian (2012) (12) showed that there was a significant difference between aloe vera and placebo groups concerning the intensity of pain, number of Analgesics and amount of bleeding, but no significant difference was observed regarding the side effects.

\section{Conclusion}

Management using anti-inflammatory drugs and analgesics is often an unsatisfactory answer due to side effects and patient compliance issues. The results of this study suggested that the aloe vera gel pill as a novel herbal pain killer represents an effective treatment for the menstrual pain with no important side effects, although further clinical trials are recommended to look at the possible side effects in an extended spectrum of subjects. The study showed that herbal pills are effective for relieving the severity of pain also. Therefore, health-care providers should consider it as treatment for young women with primary dysmenorrhoea problem. Additionally, future studies are recommended to test the feasibility and effectiveness of this kind of herbal therapy in large segments of population for more explanation.

\section{Conflict of interests}

There is no known conflict of interests associated with this paper and there has been no significant financial support for this work that could have influenced its outcome.

\section{References}

1. Andersch B, Milsom I.1982.An epidemiologic study of young women with dysmenorrhea. Am J Obstet Gynecol.144:655-660.

2. Brosens I, Puttemans P, Benagiano G.2013. Endometriosis: a life cycle approach? Am J Obstet Gynecol.209: 307-316.

3. Coco AS.1999.Primary dysmenorrhea. Am Fam Physician.60:489-498.
4. Dambhare DG, Wagh SV, Dudhe JY. 2012.Age at menarche and menstrual cycle pattern among school adolescent girls in Central India. Global journal of health science.4 (1):105-11.

5. Davis GD, Thillet E, Lindemann J.1993.Clinical characteristics of adolescent endometriosis. J Adolesc Health.14: 362- 368.

6. Demir SC, Kadayyfcy TO, Vardar MA. 2000.Dysfunctional uterine bleeding and other menstrual problems of secondary school students in Adana, Turkey. J Pediatr Adolesc Gynecol.13:171.

7. Egnew TR.2005.The meaning of healing, transcending suffering. Annals of Family Medicine. 3:255-62

8. Engstrom $\mathrm{T}$, Bratholm $\mathrm{P}$, Vilhardt $\mathrm{H}$ and Christensen N. J.1997. Effect of pregnancy on rat myometrial 2-adrenoceptor mRNA and isoproterenol-induced relaxation of isolated uterine strips. Journal of Endocrinology 153(3):393-9.

9. Harada T. 2013. Dysmenorrhea and endometriosis in young women. Yonago acta medica, 56(4).81-4.

10. Kashanian M, Moradi Lackeh M, Noori Sh.2010.Evaluation of the effect of vitamin E for pelvic pain reduction in women suffering from primary dysmenorrhea. Razi Journal of Medical Sciences.74 (17):67-74.

11. Karimi P.2008.Aloe vera, century plant, plant of immortality Persian. Journal of Farm Animal cultivation.115:58-59.

12. Khazaiyan S, Navidian A, Navvabi Rigi Sh.D.2012.The effect of oral Aloe vera gel on the intensity of primary dysmenorrhea. MedicalSurgical Nursing Journal.1 (1):49-54.

13. Ozgoli G, Goli M, Moattar F.2009.Comparison of Effects of Ginger, Mefenamic Acid, and Ibuprofen on Pain in Women with Primary Dysmenorrhea. J Altern Complement Med, 15(2), 129-32

14. Rees MC.1989. Heavy painful periods. Balliere's Clin Obstet Gynacol.3: 341-356.

15. Roberts SC, Hodgkiss C, Dibenedetto A, Lee EC.2012.Managing dysmenorrhea in young women. Nurse Practitioner.37 (7): 47-52.

16. Sakhavat L, KarimZade Meybodi MA. 2005.Comparison of Vitamin B and Ibuprofen in treatment of primary dysmenorrhea in young girls in Yazd city. Journal of Medical Sciences 
and Health Services- Yazd Shahid Sadooghi.1 (13):47-51.

17. Sundell G, Milsom I, Andersch B.1990.Factors influencing the prevalence and severity of dysmenorrhea in young women. Br J Obstet Gynaecol.97:588-594.

18. Surjushe A, Vasani R, Saple DG. 2008.Aloe vera: A short review. Indian J Dermatol. 53:163-66.
19. Vogler BK.1999.Aloe vera, a systematic review of its clinical effectiveness. $\mathrm{Br} \quad \mathrm{J}$ Gen Pract.49:823-28

20. Zahradnik HP, Hanjalic-Beck A, Groth K.2010.Nonsteroidal anti-inflammatory drugs and hormonal contraceptives for pain relief from dysmenorrhea. A review. Contraception.81:185-196.

21. Zargari, 2001. Medical plants. Second edition. Tehran University Press.

Table 1. Comparison of characteristics between experimental groups

\begin{tabular}{|c|c|c|c|c|}
\hline Characteristic & Group & $\mathrm{n}$ & Mean \pm SD & P-Value \\
\hline \multirow{2}{*}{ Age } & Aloe vera gel pill & 65 & $22.1 \pm 1.4$ & n.s \\
\cline { 2 - 5 } & Ibuprofen & 42 & $20.4 \pm 1.2$ & n.s \\
\hline \multirow{2}{*}{ Age incidence of dysmenorrhea } & Aloe vera gel pill & 65 & $15.6 \pm 1.7$ & n.s \\
\cline { 2 - 5 } & Ibuprofen & 42 & $15.1 \pm 1.8$ & n.s \\
\hline \multirow{2}{*}{ Duration of cycle } & Aloe vera gel pill & 65 & $28.2 \pm 1.6$ & n.s \\
\cline { 2 - 5 } & Ibuprofen & 42 & $27.1 \pm 1.6$ & n.s \\
\hline \multirow{2}{*}{ Duration of menstrual flow } & Aloe vera gel pill & 65 & $5.1 \pm 1.7$ & n.s \\
\cline { 2 - 5 } & Ibuprofen & 42 & $4.9 \pm 1.5$ & n.s \\
\hline Pain duration & Aloe vera gel pill & 65 & $3.5 \pm 0.9$ & n.s \\
\cline { 2 - 5 } & Ibuprofen & 42 & $3.2 \pm 0.6$ & n.s \\
\hline
\end{tabular}

*SD $=$ Standard deviations; $\mathrm{n} . \mathrm{s}=* *$ no significant.

Table2. Comparison of pain relief between different groups before intervention

\begin{tabular}{|c|c|c|c|c|c|}
\hline & \multicolumn{2}{|c|}{ Aloe Vera gel pill } & \multicolumn{2}{|c|}{ Ibuprofen } & P-Value \\
\hline Treatments & $\mathrm{n}$ & percentage & $\mathrm{n}$ & percentage & \\
& 40 & 40.3 & 31 & 27.5 & \multirow{2}{*}{0.865} \\
\hline Chemical medicine & 15 & 12.5 & 14 & 15.4 \\
\hline Herbal medicine & 15 & 10.4 & 10 & 7.5 & \\
\hline Others treatments & 70 & 63.2 & 55 & 45.4 & \\
\hline Total & \multicolumn{2}{|l}{} \\
\hline
\end{tabular}

Table 3. Comparison of pain duration in aloe vera gel and Ibuprofen groups at various time points following intervention.

\begin{tabular}{|c|c|c|c|}
\hline \multirow{2}{*}{ Treatments } & Aloe vera gel pill & Ibuprofen & \multirow{2}{*}{ Total \% } \\
\cline { 2 - 3 } & $\mathrm{n} \%$ & $\mathrm{n} \%$ & 23 \\
& 13 & 10 & $(18.5)$ \\
\hline Less than $0.5 \mathrm{~h}$ & $(19)$ & $(18.1)$ & 39 \\
& 22 & 17 & $(46)$ \\
\hline $0.5-1 \mathrm{~h}$ & $(44.5)$ & $(49)$ & 28 \\
& 18 & $(29)$ & $(36)$ \\
\hline Several hours & $(34)$ & 37 & 90 \\
& 43 & $(100)$ & $(100)$ \\
\hline
\end{tabular}

